\title{
Proton structure and parton distribution functions from HERA
}

\author{
Vladimir Chekelian ${ }^{1}$. On behalf of the $\mathrm{H} 1$ and ZEUS Collaborations. \\ ${ }^{1}$ Max Planck Institute for Physics, Munich.
}

\begin{abstract}
The H1 and ZEUS collaborations at the electron-proton collider HERA collected $e^{ \pm} p$ scattering data corresponding to an integrated luminosity of about $1 \mathrm{fb}^{-1}$. The data were taken at proton beam energies of $920,820,575$ and $460 \mathrm{GeV}$ and an electron beam energy of $27.5 \mathrm{GeV}$, with different electric charges and longitudinal polarisation of the electron beam. Using these data inclusive neutral and charged current deep inelastic cross sections were measured over six orders of magnitude in negative four-momentumtransfer squared, $Q^{2}$, and Bjorken $x$. A combination of all inclusive cross sections, published by the H1 and ZEUS collaborations at HERA, was performed. Using these combined HERA data and the individual $\mathrm{H} 1$ and ZEUS data taken using the polarised electron beams, the proton structure functions $F_{2}, F_{2}^{\gamma Z}, x F_{3}^{\gamma Z}$ and $F_{L}$ were obtained, and scaling violations, electroweak unification, and polarisation effects in the charged current process were demonstrated. The combined cross sections were used as a sole input to QCD analyses at leading, next-to-leading and next-to-next-to-leading orders, providing a new set of parton distribution functions, denoted as HERAPDF2.0. An extension of the analysis by including HERA data on charm and jet production allowed a simultaneous determination of parton distributions and the strong coupling.
\end{abstract}

\section{Introduction}

Deep inelastic scattering (DIS) of electrons ${ }^{1}$ on protons at the electron-proton collider HERA has been central to the exploration of proton structure and quark-gluon dynamics as described by perturbative Quantum Chromodynamics (pQCD). HERA was operated in two phases: HERA I, from 1992 to 2000, and HERA II, from 2002 to 2007, with an electron beam energy of $E_{e}=27.5 \mathrm{GeV}$. During HERA I and II running, the H1 and ZEUS collaborations collected total integrated luminosities of approximately $500 \mathrm{pb}^{-1}$ each, shared about equally between $e^{+} p$ and $e^{-} p$ scattering. This includes data taken with proton beam energies of $E_{p}=920,820,575$ and $460 \mathrm{GeV}$ corresponding to centre-ofmass energies of $\sqrt{s}=318,300,251$ and $225 \mathrm{GeV}$. During the HERA II period, the electron beam was longitudinally polarised with left-handed and right-handed polarisations in about equal proportion. For this period, the H1 and ZEUS collaborations published both polarised cross sections, measured using data with the longitudinally polarised electron beams, and unpolarised cross sections, obtained using data with both polarisations taken together and corrected for small residual polarisation.

The combination of all $\mathrm{H} 1$ and ZEUS inclusive neutral (NC) and charged current (CC) unpolarised deep inelastic $e^{ \pm} p$ cross section data was performed [1]. The correlated systematic uncertainties and

\footnotetext{
${ }^{1}$ The word "electron" in the paper refers to both electrons and positrons, unless otherwise stated.
} 
global normalisations were treated such that one coherent HERA data set was obtained. The results published in [1] comprise the core of this paper.

Using these combined unpolarised HERA I and HERA II and polarised HERA II cross section data, the proton structure functions $F_{2}, F_{2}^{\gamma Z}, x F_{3}^{\gamma Z}$ and $F_{L}$ were obtained, and scaling violations, electroweak unification as well as polarisation effects in $\mathrm{CC}$ were demonstrated.

The dependences of the combined NC and CC inclusive DIS cross sections from HERA on Bjorken $x$ and negative four-momentum-transfer squared, $Q^{2}$, were analysed in the pQCD framework to determine a new set of quark and gluon momentum distributions in the proton, denoted as HERAPDF2.0. An inclusion of the HERA data on charm and jet production to the analysis allowed a simultaneous determination of the parton distributions and the strong coupling.

\section{$2 \mathrm{NC}$ and $\mathrm{CC}$ cross sections and parton distributions}

The neutral current $e^{ \pm} p$ deep inelastic scattering cross section, after correction for QED radiative effects, can be written in the reduced form as

$$
\sigma_{r, N C}^{ \pm}=\frac{\mathrm{d}^{2} \sigma_{\mathrm{NC}}^{\mathrm{e}^{ \pm} \mathrm{p}}}{\mathrm{dxdQ}} \frac{x Q^{4}}{2 \pi \alpha^{2}} \frac{1}{Y_{+}}=\tilde{F}_{2} \mp \frac{Y_{-}}{Y_{+}} x \tilde{F}_{3}-\frac{y^{2}}{Y_{+}} \tilde{F}_{L} .
$$

Here, $x$ is the Bjorken scaling variable, and $y$ characterizes the inelasticity of the interaction. The helicity dependences are contained in $Y_{ \pm}=1 \pm(1-y)^{2}$. The generalised proton structure functions, $\tilde{F}_{2,3}$, occurring in Eq. 1, may be written as linear combinations of the hadronic structure functions $F_{2}, F_{2,3}^{\gamma Z}$, and $F_{2,3}^{Z}$, containing information on the QCD parton dynamics as well as the electroweak couplings of the quarks to the neutral vector bosons. The function $F_{2}$ is associated with pure photon exchange terms, $F_{2,3}^{\gamma Z}$ correspond to photon- $Z$ interference and $F_{2,3}^{Z}$ correspond to pure $Z$ exchange terms. The longitudinal structure function $\tilde{F}_{L}$ may be similarly decomposed. $\tilde{F}_{L}$ is important only at high $y$ and is expected to be negligible at large $x$ and $Q^{2}$.

The generalised proton structure functions depend on the electric charge of the lepton beam and on the lepton beam polarisation ${ }^{2}, P$, on the $Z$-boson mass $M_{Z}$ and on $\sin ^{2} \theta_{W}$, with $\theta_{W}$ being the electroweak mixing angle.

$$
\begin{aligned}
\tilde{F}_{2}^{ \pm} & =F_{2}+\kappa\left(-v_{e} \mp P a_{e}\right) F_{2}^{\gamma Z}+\kappa^{2}\left(v_{e}^{2}+a_{e}^{2} \pm 2 P v_{e} a_{e}\right) F_{2}^{Z}, \\
x \tilde{F}_{3}^{ \pm} & =\kappa\left(-a_{e} \mp P v_{e}\right) x F_{3}^{\gamma Z}+\kappa^{2}\left(2 v_{e} a_{e} \pm P\left(v_{e}^{2}+a_{e}^{2}\right)\right) x F_{3}^{Z} .
\end{aligned}
$$

Here, $\kappa=\left(4 \sin ^{2} \theta_{W} \cos ^{2} \theta_{W}\right)^{-1} Q^{2} /\left(Q^{2}+M_{Z}^{2}\right)$ determines the relative amount of $Z$ to $\gamma$ exchange. The quantities $v_{e}=-1 / 2+2 \sin ^{2} \theta_{W}$ and $a_{e}=-1 / 2$ are the vector and axial-vector couplings of the electron to the Z-boson in the Standard Model. At leading order in QCD the hadronic structure functions are related to linear combinations of sums and differences of the quark and anti-quark momentum distributions $x q\left(x, Q^{2}\right)$ and $x \bar{q}\left(x, Q^{2}\right)$ of the proton:

$$
\begin{aligned}
\left(F_{2}, F_{2}^{\gamma Z}, F_{2}^{Z}\right) & =x \sum\left(e_{q}^{2}, 2 e_{q} v_{q}, v_{q}^{2}+a_{q}^{2}\right)(q+\bar{q}), \\
\left(x F_{3}^{\gamma Z}, x F_{3}^{Z}\right) & =2 x \sum\left(e_{q} a_{q}, v_{q} a_{q}\right)(q-\bar{q}),
\end{aligned}
$$

where $v_{q}$ and $a_{q}$ are the vector and axial-vector couplings of the light quarks to the $Z$-boson, and $e_{q}$ is the charge of the quark of flavor $q$.

\footnotetext{
${ }^{2}$ The longitudinal polarisation is defined as $P=\left(N_{R}-N_{L}\right) /\left(N_{R}+N_{L}\right)$ with $N_{R}\left(N_{L}\right)$ being the number of right-handed (left-handed) leptons in the beam.
} 
The charged current $e^{ \pm} p$ cross section of longitudinally polarised leptons and unpolarised protons can be expressed in the reduced form as

$$
\tilde{\sigma}_{\mathrm{r}, \mathrm{CC}}^{ \pm} \equiv \frac{2 \pi x}{G_{F}^{2}}\left[\frac{M_{W}^{2}+Q^{2}}{M_{W}^{2}}\right]^{2} \frac{\mathrm{d}^{2} \sigma_{\mathrm{CC}}^{e^{ \pm} p}}{\mathrm{~d} x \mathrm{~d} Q^{2}}=\frac{1}{2}(1 \pm P)\left(Y_{+} W_{2}^{ \pm} \mp Y_{-} x W_{3}^{ \pm}-y^{2} W_{L}^{ \pm}\right),
$$

where $G_{F}$ is the Fermi constant defined using the weak boson masses $M_{W}$ and $M_{Z}$. This equation shows that the cross section has a linear dependence on the longitudinal polarisation of the lepton beam. In the QPM $W_{L}^{ \pm} \equiv 0$, and the structure functions $W_{2}^{ \pm}$and $x W_{3}^{ \pm}$are expressed as the flavordependent sum and difference of the quark and anti-quark momentum distributions. Only positively charged quarks contribute to $W^{-}$mediated scattering, and only negatively charged quarks couple to the exchanged $W^{+}$boson: $W_{2}^{-}=x(U+\bar{D}), \quad W_{2}^{+}=x(\bar{U}+D), \quad x W_{3}^{-}=x(U-\bar{D}), \quad x W_{3}^{+}=x(D-\bar{U})$. Below the $b$-quark mass threshold, the $U, \bar{U}$ and $D, \bar{D}$ distributions represent the sums of up-type and down-type quark densities: $U=u+c, \bar{U}=\bar{u}+\bar{c}, D=d+s, \bar{D}=\bar{d}+\bar{s}$.

The combination of NC and CC measurements makes it possible to determine both the valencequark distributions, $x u_{v}=x(U-\bar{U})$ and $x d_{v}=x(D-\bar{D})$, and the sea-quark distributions, $x \bar{U}$ and $x \bar{D}$. The parton distribution functions (PDFs) are determined by a fit to the $x$ and $Q^{2}$ dependence of data using the linear DGLAP equations [2] at leading order (LO), next-to-leading order (NLO) or next-tonext-to-leading order (NNLO) in $\mathrm{pQCD}$. The experimentally measured scaling violations determine the gluon distribution in the fit.

\section{Combination of all inclusive NC and CC cross section data at HERA}

In order to combine all inclusive unpolarised $\mathrm{NC}$ and $\mathrm{CC}$ cross section measurements published by H1 and ZEUS, they were translated onto common $x, Q^{2}$ grids and averaged [1]. In total 41 data sets from $\mathrm{H} 1$ and ZEUS entered into this combination. The references to individual data sets can be found in [1]. Separate measurements from the same data set were not translated to the same grid point and the vast majority of grid points accumulated data from both $\mathrm{H} 1$ and ZEUS measurements; the typical case is six measurements from six different data sets. The translation of the original measurements to the nearest grid points was performed using results of the QCD and fractal fits to the combined data; for details see [1]. The procedure applied for the combination follows very closely to the one used for the previously published combination of the HERA I data [3] and for the combination of the HERA I data and preliminary HERA II results [4].

\subsection{Averaging cross sections}

The averaging of the data points was performed using the HERAverager [5] tool which is based on a $\chi^{2}$ minimisation method [6]. This method imposes that there is one and only one correct value for the cross section of each process at each point of the phase space. These values are estimated by optimising a vector, $m^{i}$, which is the result of the averaging for the cross sections. The $\chi^{2}$ definition used takes into account the correlated and uncorrelated systematic uncertainties of the H1 and ZEUS crosssection measurements and allows for shifts of the data to accommodate the correlated uncertainties. The $\chi^{2}$ function involves all measurements from all data sets and can be approximated by

$$
\chi_{\text {tot }}^{2} \approx \chi_{\min }^{2}+\sum_{i=1, N_{M}} \frac{\left[m^{i}-\sum_{j} \gamma_{j}^{i} m^{i} b_{j}^{\prime}-\mu^{i}\right]^{2}}{\delta_{i, \mathrm{stat}}^{2} \mu^{i}\left(m^{i}-\sum_{j} \gamma_{j}^{i} m^{i} b_{j}^{\prime}\right)+\left(\delta_{i, \text { uncor }} m^{i}\right)^{2}}+\sum_{j}\left(b_{j}^{\prime}\right)^{2},
$$

where $\chi_{\min }^{2}$ is the minimum of $\chi_{\text {tot }}^{2}, N_{M}$ is the number of combined measurements, $\mu^{i}$ is the average value at point $i$, and $\gamma_{j}^{i}, \delta_{i \text {,stat }}$ and $\delta_{i \text {,uncor }}$ are its relative correlated systematic, relative statistical 

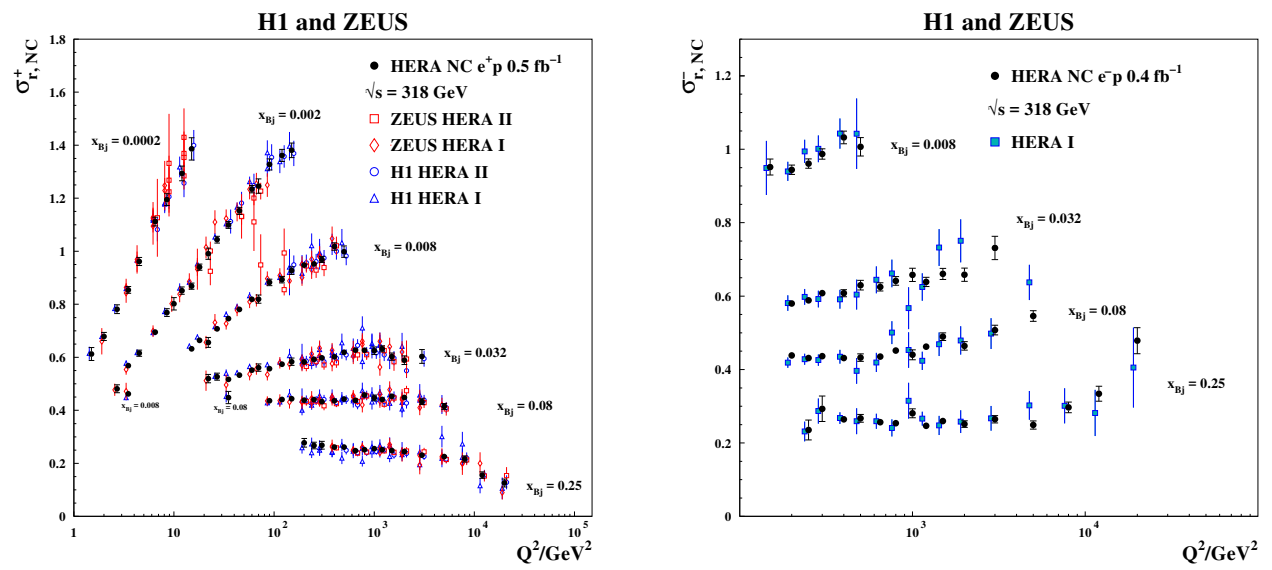

Figure 1. Left: The combined HERA data for the inclusive NC $e^{+} p$ reduced cross sections as a function of $Q^{2}$ for six selected values of $x$, compared to the individual $\mathrm{H} 1$ and ZEUS data. Right: The combined HERA data for the inclusive $\mathrm{NC} e^{-} p$ reduced cross section as a function of $Q^{2}$ for four selected values of $x$, compared to the previously published combined results from HERA I alone.

and relative uncorrelated systematic uncertainties, respectively. The shifts $b_{j}^{\prime}$, also called nuisance parameters, are related to the original correlated shifts $b_{j}$ of the cross sections in units of sigma of the respective correlated systematic uncertainties through an orthogonal transformation, which is also used to determine $\gamma_{j}^{i}$ [3]. The average values $\mu^{i}$ and systematic shifts $b_{j}$ are determined analytically from a system of linear equations $\partial \chi_{\text {tot }}^{2} / \partial m^{i}=0$ and $\partial \chi_{\text {tot }}^{2} / \partial b_{j}=0$. An iterative procedure is used to determine the average of the data. It converges after two iterations.

\subsection{Combined neutral and charged current cross sections at HERA}

The 2927 published cross sections from the 41 data sets were combined to become 1307 combined cross-section measurements. For the resulting 1620 degrees of freedom, a $\chi_{\min }^{2}=1687$ was obtained, indicating consistency of the input data. It was also checked that the pull distributions for the different data sets and in different corners of the phase space showed good consistency of all data.

In Fig. 1 (left) the individual H1 and ZEUS and the HERA combined reduced cross sections for NC $e^{+} p$ scattering at $\sqrt{s}=318 \mathrm{GeV}$ are shown as a function of $Q^{2}$ for selected values of $x$. The improvement due to combination is clearly visible. In Fig. 1 (right) a comparison between the combined HERA data and the combination of HERA I data alone [3] for NC $e^{-} p$ scattering is shown. The integrated luminosity for $e^{-} p$ scattering was very limited for the HERA I period, therefore, the improvements due to the new combination are even more substantial than for $e^{+} p$ scattering.

The combined reduced cross sections for $\mathrm{NC}$ and $\mathrm{CC} e^{ \pm} p$ scattering are obtained together with their statistical, uncorrelated and correlated systematic uncertainties. At the proton beam energy of $E_{p}=920 \mathrm{GeV}$, corresponding to the highest centre-of-mass energy of $\sqrt{s}=318 \mathrm{GeV}$, they are shown in Fig. 2 for NC (left) and CC (right) as a function of $Q^{2}$ for different values of $x$. The total uncertainties for NC are below $1.5 \%$ over the $Q^{2}$ range of $3 \leq Q^{2} \leq 500 \mathrm{GeV}^{2}$ and below $3 \%$ up to $Q^{2}=3000 \mathrm{GeV}^{2}$. Cross sections are provided for values of $Q^{2}$ between $Q^{2}=0.045 \mathrm{GeV}^{2}$ and $Q^{2}=50000 \mathrm{GeV}^{2}$ and for values of $x$ between $x=6 \times 10^{-7}$ and $x=0.65$. 

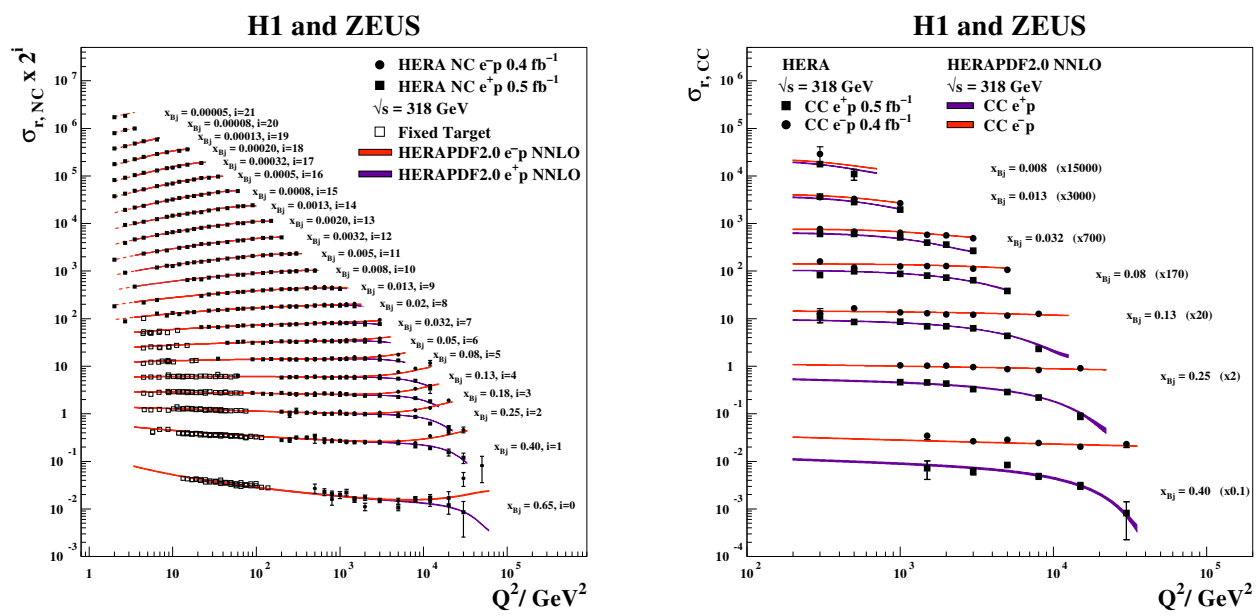

Figure 2. Left (Right): The combined HERA data for the inclusive NC (CC) $e^{+} p$ and $e^{-} p$ reduced cross sections at $\sqrt{s}=318 \mathrm{GeV}$ together with fixed-target data and the predictions of HERAPDF2.0 NNLO. The bands represent the total uncertainties on the predictions.

The combined $\mathrm{NC}$ and $\mathrm{CC}$ cross sections were integrated to obtain the single-differential cross sections $\mathrm{d} \sigma / \mathrm{d} Q^{2}$. The integration over $x$ was performed in the region $y<0.9$, using the shapes as predicted from HERAPDF2.0. All correlated and uncorrelated uncertainties were taken into account. The cross-sections $\mathrm{d} \sigma / \mathrm{d} Q^{2}$ are shown in Fig. 3 (left) for NC and CC $e^{-} p$ and $e^{+} p$ scattering together with predictions from HERAPDF2.0 NLO. Whereas the NC cross sections are three orders of magnitude larger at low $Q^{2} \approx 100 \mathrm{GeV}^{2}$, where they are dominated by virtual photon exchange, the NC and $\mathrm{CC}$ cross sections become similar in magnitude at $Q^{2} \approx 10000 \mathrm{GeV}^{2}$, i.e. at around the massscale squared of the electroweak bosons, demonstrating the success of electroweak unification in the Standard Model.

The total CC cross sections $\sigma_{\mathrm{CC}}^{e^{ \pm} p}$ measured by $\mathrm{H} 1$ and ZEUS in the range $Q^{2}>400 \mathrm{GeV}^{2}$ and $y<0.9$ are shown in Fig. 3 (right) as a function of the electron beam polarisation. The cross sections were obtained using the HERA II CC data with left-handed and right-handed electron beam polarisations [7, 8] and using CC data from HERA I with unpolarised electron beams [9]. The measurements are compared to expectations of the Standard Model based on the HERAPDF1.5[4] parameterisation of the parton densities of the proton. The measurements exhibit the expected linear dependence as a function of the polarisation and are consistent with the axiomatic absence of right-handed charged currents in the Standard Model.

\subsection{Scaling violations of $F_{2}$ and the proton structure function $F_{2}^{\gamma Z}$}

The dominant contribution to the NC cross section is due to $\tilde{F}_{2}$, see Eqs. 1-3. Therefore, the combined reduced NC cross sections in Fig. 2 (left) reveal the main properties of the proton structure function $\tilde{F}_{2}$. At $x \simeq 0.1$ they are independent of $Q^{2}$ demonstrating scaling of $\tilde{F}_{2}$ in this region. At higher and lower values of $x$ one observes scaling violations due to emission of gluons from quarks and splitting of gluons into quark-antiquark pairs, respectively. Values of the structure function $\tilde{F}_{2}$ determined 

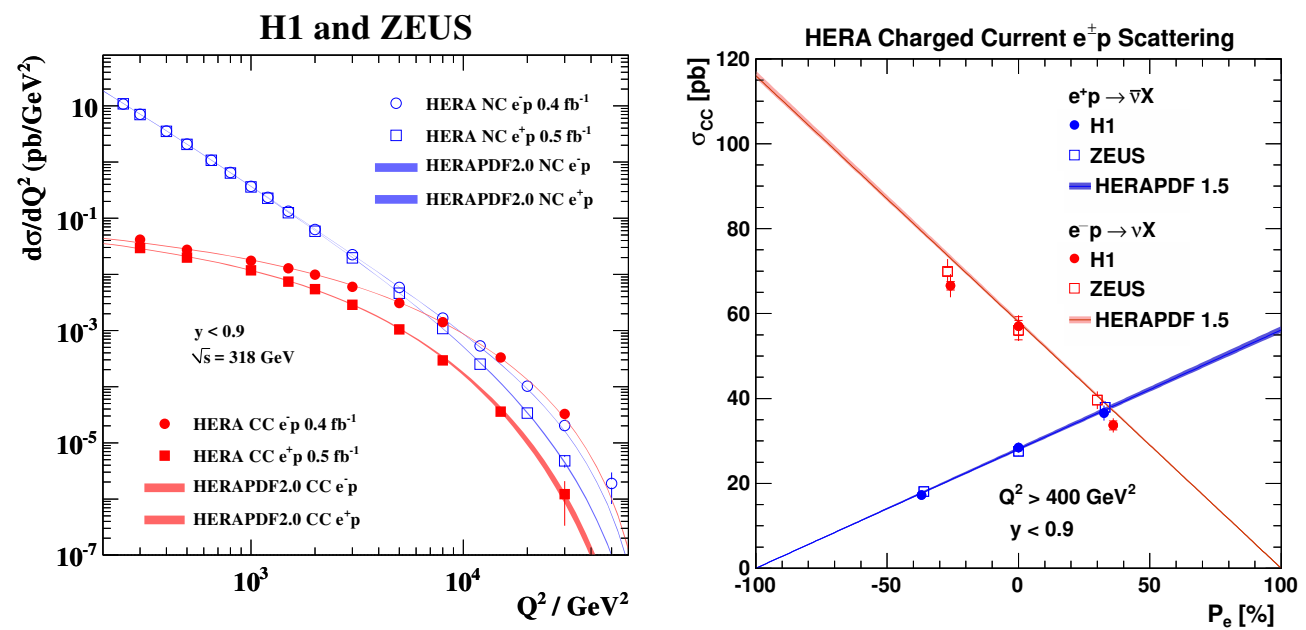

Figure 3. Left: The combined HERA NC and CC $e^{-} p$ and $e^{+} p$ cross sections, $\mathrm{d} \sigma / \mathrm{d} Q^{2}$, together with predictions from HERAPDF2.0 NLO. Right: The dependence of the total $e^{+} p$ and $e^{-} p$ CC cross sections in the region $Q^{2}>400 \mathrm{GeV}^{2}$ and $y<0.9$ on the electron beam polarisation $P_{e}$, together with the Standard Model predictions based on HERAPDF1.5.

from the reduced cross sections in the phase space, where corrections due to both $x \tilde{F}_{3}$ and $\tilde{F}_{\mathrm{L}}$ are small, are displayed as a function of $x$ at fixed $Q^{2}$ in Fig. 4 (left) together with HERAPDF2.0 NLO predictions. Here, the scaling violations manifest themselves by the rise towards low values of $x$, which is becoming steeper as $Q^{2}$ increases.

The polarisation effects in NC allow the parity violating component of the proton structure function $\tilde{F}_{2}$, namely $F_{2}^{\gamma Z}$, to be determined. For a given electron charge the difference of the left $(\mathrm{L})$ and right $(\mathrm{R})$ polarised $\mathrm{NC}$ cross sections can be written as

$$
\frac{\sigma_{r, N C}^{ \pm}\left(P_{L}^{ \pm}\right)-\sigma_{r, N C}^{ \pm}\left(P_{R}^{ \pm}\right)}{P_{L}^{ \pm}-P_{R}^{ \pm}}=\kappa\left[\mp a_{e} F_{2}^{\gamma Z}+\frac{Y_{-}}{Y_{+}} v_{e} x F_{3}^{\gamma Z}-\frac{Y_{-}}{Y_{+}} \kappa\left(v_{e}^{2}+a_{e}^{2}\right) x F_{3}^{Z}\right] .
$$

Subtracting the differences for the $e^{-} p$ and $e^{+} p$ interactions, the terms proportional to $x F_{3}^{\gamma Z}$ and $x F_{3}^{Z}$ cancel, and $F_{2}^{\gamma Z}$ can be directly extracted from the cross section data. H1 performed such measurements [7] for $Q^{2} \geq 200 \mathrm{GeV}^{2}$ using HERA II data with the polarised electron beams. Only a weak $Q^{2}$ dependence is expected, and therefore the measurements were transformed to a common $Q^{2}$ value of $1500 \mathrm{GeV}^{2}$ using the H1PDF 2012 fit [7] and were averaged in each $x$ bin. The parity violating structure function $F_{2}^{\gamma Z}$ measured for the first time is displayed in Fig. 4 (right).

\subsection{Electron charge dependence of the NC cross section and determination of $x F_{3}^{\gamma Z}$}

The different charges of electrons and positron beams allow the structure function $x \tilde{F}_{3}$ to be measured due to difference in the sign in front of the $x \tilde{F}_{3}$ contribution in Eq. 1 . The differences in the combined $e^{+} p$ and $e^{-} p$ NC reduced cross sections are clearly visible at high $Q^{2}$ in Fig. 2 (left). The structure function $x \tilde{F}_{3}$ was extracted by subtracting the combined $\mathrm{NC} e^{+} p$ from the combined $\mathrm{NC} e^{-} p$ cross sections at HERA for $Q^{2} \geq 1000 \mathrm{GeV}^{2}$. The dominant contribution to $x \tilde{F}_{3}$ arises from the $\gamma Z$ interference, which allows $x F_{3}^{\gamma Z}$ to be determined using Eq. 2. The structure function $x F_{3}^{\gamma Z}$ is non-singlet 

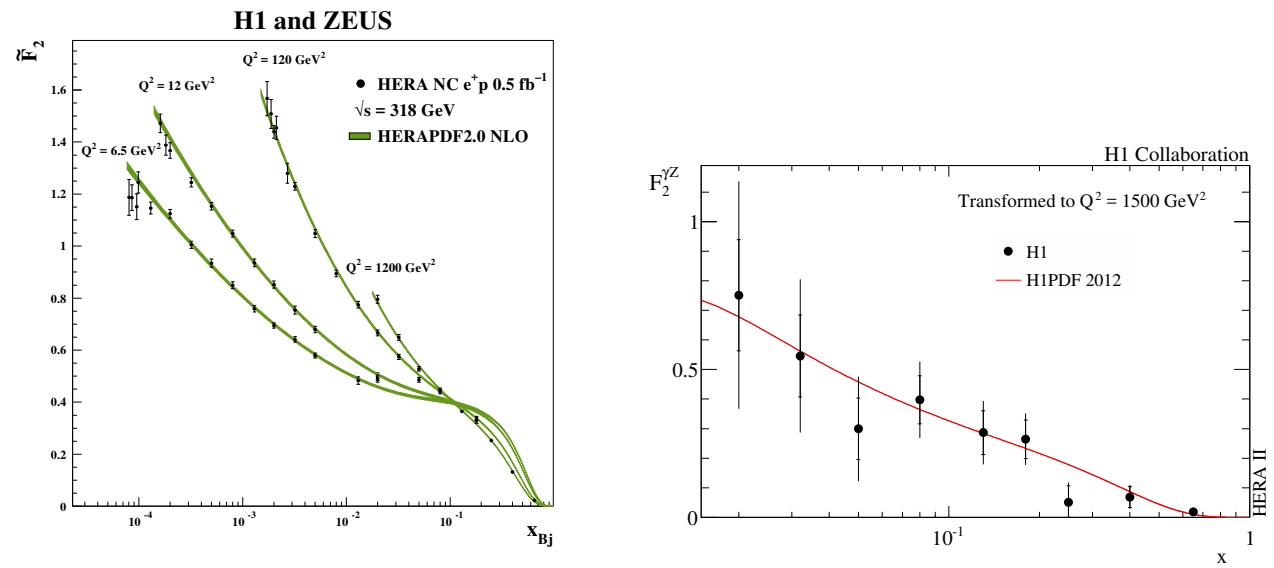

Figure 4. Left: The structure function $\tilde{F}_{2}$ as extracted from the HERA combined reduced NC cross sections for four values of $Q^{2}$ together with predictions using HERAPDF2.0 NLO. Right: The structure function $F_{2}^{\gamma Z}$ as a function of $x$ at $Q^{2}=1500 \mathrm{GeV}^{2}$ compared to predictions based on H1PDF 2012.

and shows little dependence on $Q^{2}$. The measured $x F_{3}^{\gamma Z}$ at different $Q^{2}$ values can thus be averaged taking into account the small $Q^{2}$ dependence. The averaged values of $x F_{3}^{\gamma Z}$ [1], determined for a $Q^{2}$ value of $1000 \mathrm{GeV}^{2}$, are shown in Fig. 5 (left). The measurement directly probes the valence-quark content of the proton and is well described by HERAPDF2.0 NLO.

\subsection{Measurement of the longitudinal structure function $F_{L}$}

The longitudinal structure function $F_{L}$ is obtained at HERA $[10,11]$ in a model independent way using measurements at fixed $x$ and $Q^{2}$ but different $y$. This is achieved by H1 and ZEUS using data from
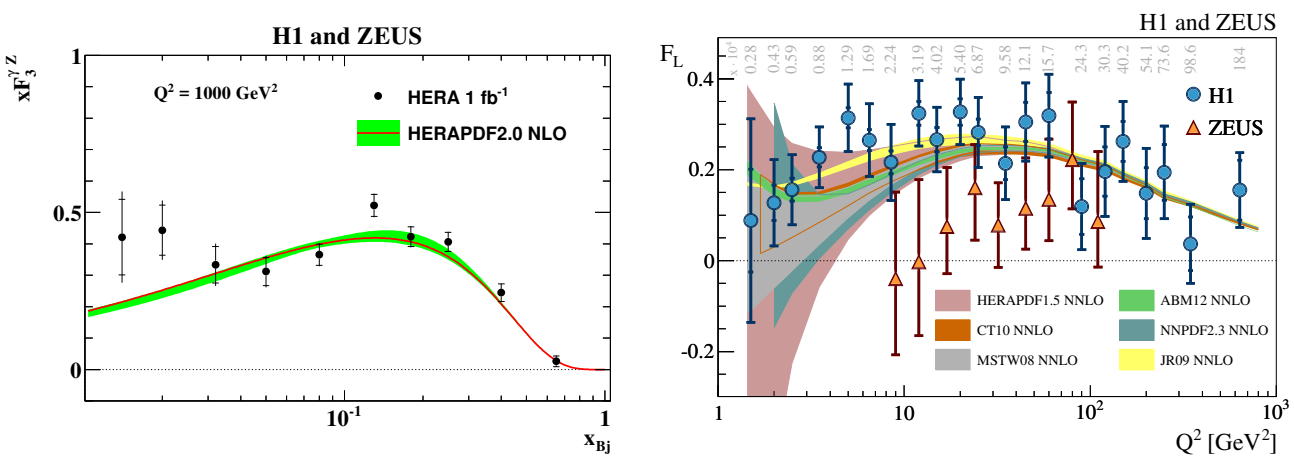

Figure 5. Left: The structure function $x F_{3}^{\gamma Z}$ for ten values of $Q^{2}$ together with predictions from HERAPDF2.0 NLO. Right: The H1 and ZEUS measurements of $F_{L}\left(Q^{2}\right)$. The resulting $x$ values of the averaged $F_{L}$ are given on top for each point in $Q^{2}$. The data are compared to NNLO predictions based on a selection of PDF sets as indicated. 
running periods with reduced $(460 \mathrm{GeV}$ and $575 \mathrm{GeV})$ and nominal $(920 \mathrm{GeV})$ proton beam energies. According to Eq. 1, the $F_{L}$ contribution to the reduced cross section is proportional to $f(y)=y^{2} /[1+$ $\left.(1-y)^{2}\right]$. Therefore, the $F_{L}$ values can be determined as slopes of straight-line fits of the measured $\sigma_{r, N C}\left(x, Q^{2}, y\right)$ values as a function of the $y$-dependent factor $f(y)$. More sophisticated procedures to determine $F_{L}$ and $F_{2}$ were applied by $\mathrm{H} 1$ and ZEUS to take into account correlations of data points due to systematic uncertainties. The $\mathrm{H} 1$ and ZEUS measurements of $F_{L}\left(x, Q^{2}\right)$ were averaged at fixed $Q^{2}$, and the resulting values of $F_{L}\left(Q^{2}\right)$ are shown in Fig. 5 (right). The H1 measurements generally lie above the ZEUS results. The differences were examined in the common $Q^{2}$ range, $9 \leq Q^{2} \leq 110 \mathrm{GeV}^{2}$. Taking into account both the correlations between the $\mathrm{H} 1$ and between the ZEUS measurements, a $\chi^{2}$ of 11 was obtained for 8 degrees of freedom. This corresponds to a pvalue of $20 \%$, indicating consistency of the $\mathrm{H} 1$ and ZEUS $F_{L}$ results. The data are reasonably well reproduced by theoretical predictions at NNLO. The measurements of $F_{L}$ were used to determine the ratio $R=F_{L} /\left(F_{2}-F_{L}\right)$. For $1.5 \leq Q^{2} \leq 800 \mathrm{GeV}^{2}$ the ratio $R$ measured by $\mathrm{H} 1$ showed constant behaviour yielding the averaged value of $R=0.23 \pm 0.04$ [10]. The corresponding ZEUS result is $R=0.105_{-0.037}^{+0.055}[11]$ in the range $9 \leq Q^{2} \leq 110 \mathrm{GeV}^{2}$.

\section{QCD analysis of the combined inclusive cross sections at HERA}

The combined inclusive $e^{ \pm} p$ NC and CC cross section data [1] were used as the sole input in the pQCD analysis, resulting in the PDF set called HERAPDF2.0 [1]. This analysis was performed within the HERAFitter framework [12] by fitting the predictions obtained by solving the DGLAP

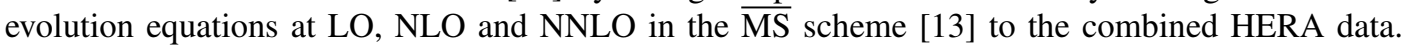
The DGLAP equations yield the PDFs at all scales $\mu_{\mathrm{f}}^{2}$ and $x$, if they are provided as functions of $x$ at some starting scale. In variable-flavour-number schemes, this scale has to be below the charm-quark mass parameter, $M_{c}$, squared. It was chosen to be $1.9 \mathrm{GeV}^{2}$. The renormalisation and factorisation scales were taken to be $Q^{2}$. The heavy-quark coefficient functions were calculated in the generalmass variable-flavour-number scheme called RTOPT [14] for the NC structure functions. For the CC structure functions, the zero-mass approximation was used, since all HERA CC data have $Q^{2} \gg M_{b}^{2}$, where $M_{b}$ is the beauty-quark mass parameter in the calculation. The value of $M_{c}$ and $M_{b}$ were chosen after performing $\chi^{2}$ scans of NLO and NNLO pQCD fits to the combined inclusive data together with the HERA combined charm data [15] or with data on beauty production from ZEUS [16] and H1 [17]. The value of the strong coupling constant was taken to be $\alpha_{s}\left(M_{Z}^{2}\right)=0.118$ [18] at NLO and NNLO and $\alpha_{s}\left(M_{Z}^{2}\right)=0.130$ [19] for the LO fit.

In the approach of HERAPDF, the PDFs of the proton, $x f$, are generically parameterised at the starting scale as $x f(x)=A x^{B}(1-x)^{C}\left(1+D x+E x^{2}\right)$. The PDFs parameterised are the gluon distribution, $x g$, the valence-quark distributions, $x u_{v}, x d_{v}$, and the $u$-type and $d$-type anti-quark distributions, $x \bar{U}$, $x \bar{D}$. The relations $x \bar{U}=x \bar{u}$ and $x \bar{D}=x \bar{d}+x \bar{s}$ are assumed at the starting scale.

The central parameterisation is

$$
\begin{array}{ll}
x g(x)=A_{g} x^{B_{g}}(1-x)^{C_{g}}-A_{g}^{\prime} x^{B_{g}^{\prime}}(1-x)^{C_{g}^{\prime}}, & \\
x u_{v}(x)=A_{u_{v}} x^{B_{u_{v}}}(1-x)^{C_{u_{v}}}\left(1+E_{u_{v}} x^{2}\right), & x d_{v}(x)=A_{d_{v}} x^{B_{d_{v}}}(1-x)^{C_{d_{v}}}, \\
x \bar{U}(x)=A_{\bar{U}} x^{B_{\bar{U}}}(1-x)^{C_{\bar{U}}}\left(1+D_{\bar{U}} x\right), & x \bar{D}(x)=A_{\bar{D}} x^{B_{\bar{D}}}(1-x)^{C_{\bar{D}}} .
\end{array}
$$

In the gluon distribution, $x g$, an additional term of the form $A_{g}^{\prime} x^{B_{g}^{\prime}}(1-x)^{C_{g}^{\prime}}$ was subtracted at NLO and NNLO to allow more flexibility for the gluon distribution. The fits without this term are marked by adding "AG" (alternative gluon) to the fit notation, e.g. HERAPDF2.0AG LO for the fit at LO.

To safely remain in the kinematic region where pQCD is expected to be applicable, only cross sections for $Q^{2}$ starting from $Q_{\min }^{2}=3.5 \mathrm{GeV}^{2}$ were used in the analysis. In the kinematic region of the fits, target-mass corrections and large- $x$ higher-twist corrections are expected to be negligible. 

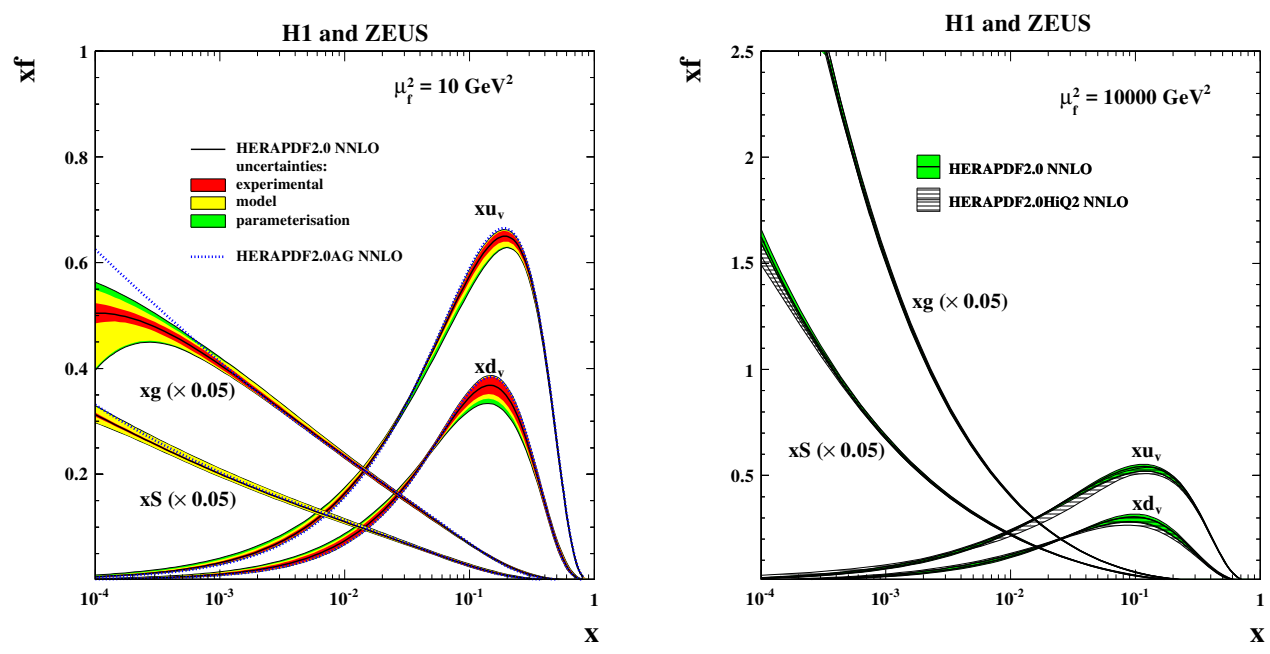

Figure 6. The parton distribution functions $x u_{v}, x d_{v}, x S=2 x(\bar{U}+\bar{D})$ and $x g$; the gluon and sea distributions are scaled down by a factor 20. Left: HERAPDF2.0 NNLO at $\mu_{\mathrm{f}}^{2}=10 \mathrm{GeV}^{2}$. The experimental, model and parameterisation uncertainties are shown. The dotted lines represent HERAPDF2.0AG NNLO with the alternative gluon parameterisation. Right: HERAPDF2.0 NNLO at $\mu_{\mathrm{f}}^{2}=10000 \mathrm{GeV}^{2}$ with $Q_{\min }^{2}=3.5 \mathrm{GeV}^{2}$ compared to HERAPDF2.0HiQ2 NNLO with $Q_{\min }^{2}=10 \mathrm{GeV}^{2}$. The bands represent the total uncertainties.

Experimental uncertainties were determined using the Hessian method with the criterion $\Delta \chi^{2}=1$, justified by the consistency of the input HERA data. In addition to experimental uncertainties, model and parameterisation uncertainties were also considered. The uncertainties on HERAPDF2.0 due to the choice of model settings and the form of the parameterisation were evaluated by varying the assumptions. Two kinds of parameterisation uncertainties were considered, the variation of the starting scale and the addition of terms with parameters $D$ and $E$; see the generic form of parameterisation above. The only significant difference from the 14-parameter central fit came from the 15-parameter fit, for which $D_{u_{v}}$ was non zero. The final parameterisation uncertainty for a given quantity is taken as the largest of the uncertainties. The total PDF uncertainty is obtained by adding in quadrature the experimental, the model and the parameterisation uncertainties. The setup used in this QCD analysis follows closely to the one established earlier for HERAPDF1.0 [3] for fitting the combined HERA I data [3].

\subsection{HERAPDF2.0 and its variants}

Using the standard settings discussed above, the HERAPDF2.0 sets of PDS were determined at NLO and NNLO. A summary of HERAPDF2.0 NNLO is shown in Fig. 6 (left) at the scale $\mu_{\mathrm{f}}^{2}=10 \mathrm{GeV}^{2}$. The experimental, model and parameterisation uncertainties are shown separately. The distribution of HERAPDF2.0AG NNLO with the alternative gluon parameterisation is shown as well. At NNLO, the gluon distribution of HERAPDF2.0 ceases to rise at low $x$ in contrast to HERAPDF2.0AG NNLO.

It was observed that the $\chi^{2}$ per degree of freedom in the fit is decreasing and getting closer to unity with increasing value of $Q_{\min }^{2}$. This continues up to $Q_{\min }^{2}=10 \mathrm{GeV}^{2}$, remaining about the same for higher values of $Q_{\min }^{2}$. This could be caused by non-perturbative contributions missing in the theory used in the fit. A question may arise whether including data from the kinematic region of low $x$ and low 
$Q^{2}$, i.e. below $10 \mathrm{GeV}^{2}$, in the PDF fits could introduce a bias on predictions for high $x$ and high $Q^{2}$. To answer this, the HERAPDF2.0HiQ2 NLO and NNLO fits were performed with $Q_{\min }^{2}=10 \mathrm{GeV}^{2}$ and the same other settings as for HERAPDF2.0. At NLO, the gluon distributions of HERAPDF2.0 and HERAPDF2.0HiQ2 were found to be compatible within uncertainties. At NNLO, the two gluon distributions differ significantly. Using the higher $Q_{\min }^{2}$ at NNLO causes the gluon distribution to turn over at low $x$. Nevertheless, it was demonstrated that at the high scale of $\mu_{\mathrm{f}}^{2}=10000 \mathrm{GeV}^{2}$, the PDFs resulting from the two fits are very similar, see Fig. 6 (right). This confirms that the value of $Q_{\text {min }}^{2}=3.5 \mathrm{GeV}^{2}$ is safe for $\mathrm{pQCD}$ fits to HERA data and no bias was introduced for applications at higher scales like cross-section predictions for the LHC.

The PDFs of HERAPDF2.0 NNLO are directly compared in Fig. 7 to the PDFs of MMHT2014 [20], for which the same heavy-flavour scheme, i.e. RTOPT, was used. Also comparisons to the PDFs of CT10 [21], for which a heavy-flavour-scheme based on ACOT was used, and NNPDF3.0 [22], for which the FONLL scheme was used, are shown. All PDFs are compatible taking into account full uncertainties. The largest relative discrepancy $(\approx 2.5 \sigma)$ is found in the shape of the $x u_{v}$ distribution at $x \approx 0.4$. The variants of the QCD fit, HERAPDF2.0FF3A and HERAPDF2.0FF3B, obtained using fixed-flavour-number schemes are compared in Fig. 8 to the PDFs of ABM11 FF(3N) [23] and NNPDF3.0 FF(3N) [22] with similar flavour schemes. The gluon distributions are quite similar. Some differences are observed in the $x u_{v}$ and $x d_{v}$ valence distributions.

An extension of the analysis by including HERA data on charm and jet production, denoted as HERAPDF2.0Jets, made a simultaneous determination of parton distributions and the strong coupling at the scale $M_{Z}^{2}$ possible. A full uncertainty analysis was performed including model and parameterisation uncertainties as well as additional hadronisation uncertainties on the jet data. The $\chi^{2}$ and the resulting PDFs of the HERAPDF2.0Jets fit are very close to the values for the fit with fixed
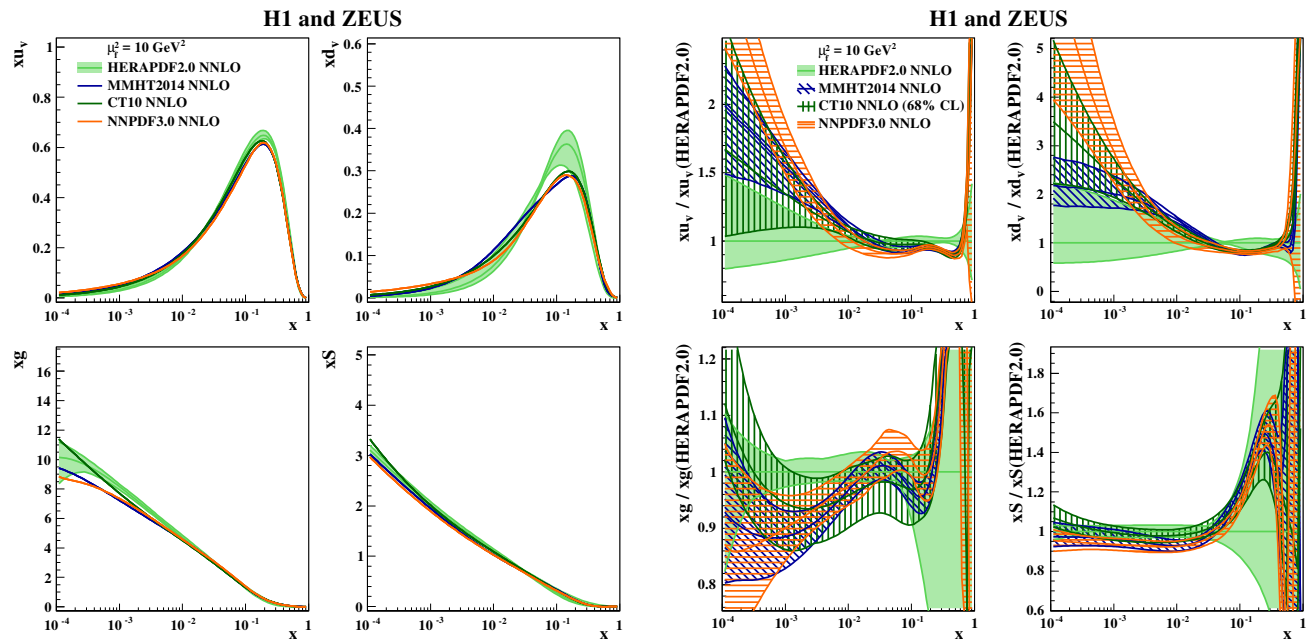

Figure 7. The parton distribution functions $x u_{v}, x d_{v}, x g$ and $x S=2 x(\bar{U}+\bar{D})$ of HERAPDF2.0 NNLO at $\mu_{\mathrm{f}}^{2}=10 \mathrm{GeV}^{2}$ compared to those of MMHT2014, CT10 and NNPDF3.0. The left panel shows the distribution with uncertainties only for HERAPDF2.0. The right panel shows the PDFs normalised to HERAPDF2.0 and with uncertainties for all PDFs. 

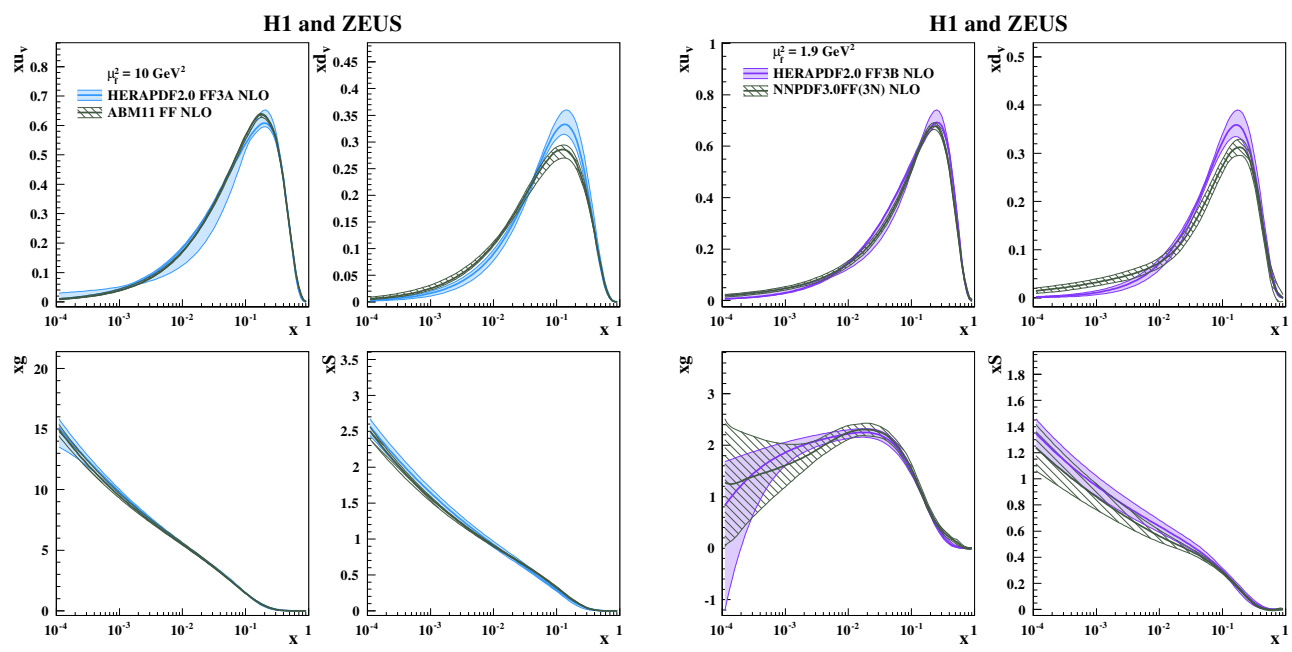

Figure 8. The parton distribution functions $x u_{v}, x d_{v}, x g$ and $x S=2 x(\bar{U}+\bar{D})$. Left: HERAPDF2.0FF3A at $\mu_{\mathrm{f}}^{2}=10 \mathrm{GeV}^{2}$ compared to ABM11 FF. Right: HERAPDF2.0FF3B at the starting scale $\mu_{\mathrm{f}_{0}}^{2}=1.9 \mathrm{GeV}^{2}$ compared to NNPDF3.0FF (3N).

$\alpha_{s}\left(M_{Z}^{2}\right)=0.118$, because the HERAPDF2.0Jets fit with free $\alpha_{s}\left(M_{Z}^{2}\right)$ yields a very similar value of

$$
\left.\alpha_{s}\left(M_{Z}^{2}\right)=0.1183 \pm 0.0009(\exp ) \pm 0.0005 \text { (model } / \text { param. }\right) \pm 0.0012 \text { (hadron.) }{ }_{-0.0030}^{+0.0037}(\text { scale })
$$

The uncertainty on $\alpha_{s}\left(M_{Z}^{2}\right)$ due to scale uncertainties was evaluated by varying the renormalisation and factorisation scales by a factor of two, both separately and simultaneously, and taking the maximal positive and negative deviations. The result on $\alpha_{s}\left(M_{Z}^{2}\right)$ is compatible with the world average [18] and it is competitive with other determinations at NLO.

\section{Summary and Conclusion}

The H1 and ZEUS collaborations measured inclusive $e^{ \pm} p$ scattering cross sections at HERA from 1994 to 2007, collecting a total integrated luminosity of about $1 \mathrm{fb}^{-1}$ at four different centre-of-mass energies, with two different detectors changing and improving over time. All inclusive data were combined to create one consistent set of $\mathrm{NC}$ and $\mathrm{CC}$ cross-section measurements for unpolarised $e^{ \pm} p$ scattering, spanning six orders of magnitude in both negative four-momentum-transfer squared, $Q^{2}$, and Bjorken $x$. Combined cross sections are provided for values of $Q^{2}$ between $Q^{2}=0.045 \mathrm{GeV}^{2}$ and $Q^{2}=50000 \mathrm{GeV}^{2}$ and values of $x$ between $x=6 \times 10^{-7}$ and $x=0.65$. They are the most precise measurements ever published for $e p$ scattering over such a large kinematic range. Using the combined HERA data and the individual data taken by $\mathrm{H} 1$ and ZEUS with longitudinal beam polarisation, the proton structure functions $F_{2}, F_{2}^{\gamma Z}, x F_{3}^{\gamma Z}$ and $F_{L}$ were obtained, and scaling violations, electroweak unification and polarisation effects in $\mathrm{CC}$ were demonstrated.

The combined inclusive cross sections were used as input to a QCD analysis within the DGLAP formalism. The resulting parton distribution functions are denoted HERAPDF2.0 and are available at LO, NLO and NNLO using variable-flavour-number scheme. Two variants, HERAPDF2.0 FF3A and FF3B, using fixed-flavour number schemes, are also available at NLO. Other variants of the fit were 
also performed exploring an alternative, positively defined, gluon parameterisation and different $Q_{\min }^{2}$ values. A measurement of the strong coupling constant was performed using a perturbative QCD fit, denoted as HERAPDF2.0Jets, for which the inclusive cross sections were augmented with selected jet- and charm-production cross section data as measured by the H1 and ZEUS collaborations.

\section{References}

[1] H. Abramowicz et al. [H1 and ZEUS Collaborations], Eur. Phys. J. C 75, 580 (2015), [arXiv:1506.06042].

[2] V. N. Gribov and L. N. Lipatov, Sov. J. Nucl. Phys. 15, 438 (1972); Sov. J. Nucl. Phys. 15, 675 (1972); L. N. Lipatov, Sov. J. Nucl. Phys. 20, 94 (1975); Y. L. Dokshitzer, Sov. Phys. JETP 46, 641 (1977); G. Altarelli and G. Parisi, Nucl. Phys. B 126, 298 (1977).

[3] F. Aaron et al. [H1 and ZEUS Collaborations], JHEP 1001, 109 (2010), [arXiv:0911.0884].

[4] HERAPDF1.5: URL https://www.desy.de/h1zeus/combined_results/herapdftable/ .

[5] The documentation can be found at: URL https://wiki-zeuthen.desy.de/HERAverager .

[6] F. Aaron et al. [H1 Collaboration], Eur. Phys. J. C 63, 625 (2009), [arXiv:0904.0929].

[7] F. Aaron et al. [H1 Collaboration], JHEP 09, 61 (2012), [arxiv:1206.7007].

[8] S. Chekanov et al. [ZEUS Collaboration], Eur. Phys. J. C 61, 223 (2009), [arXiv:0812.4620];

H. Abramowicz et al. [ZEUS Collaboration], Eur. Phys. J. C 70, 945 (2010), [arXiv:1008.3493].

[9] C. Adloff et al. [H1 Collaboration], Eur. Phys. J. C 13, 609 (2000), [hep-ex/9908059]; Eur. Phys.

J. C 19, 269 (2001), [hep-ex/0012052]; Eur. Phys. J. C 30, 1 (2003), [hep-ex/0304003];

J. Breitweg et al. [ZEUS Collaboration], Eur. Phys. J. C 12, 411 (2000), [Erratum-ibid. C 27, 305 (2003)], [hep-ex/9907010];

S. Chekanov et al. [ZEUS Collaboration], Phys. Lett. B 539, 197 (2002), [Erratum-ibid. B 552, 308 (2003)], [hep-ex/0205091]; Eur. Phys. J. C 32, 1 (2003), [hep-ex/0307043].

[10] V. Andreev et al. [H1 Collaboration], Eur. Phys. J. C 74, 2814 (2014), [arxiv:1312.4821].

[11] H. Abramowicz et al. [ZEUS Collaboration], Phys. Rev. D90, 072002 (2014), [arXiv:1404.6376].

[12] S. Alekhin et al. (2014), [arXiv:1410.4412]; Documentation at: URL http://www.herafitter.org .

[13] B. Fanchiotti, S. Kniehl and A. Sirlin, Phys. Rev. D 48, 307 (1993), [hep-ph/9212285].

[14] R. S. Thorne and R. G. Roberts, Phys. Rev. D 57, 6871 (1998), [hep-ph/9709442];

R. S. Thorne, Phys. Rev. D 73, 054019 (2006), [hep-ph/0601245];

R. S. Thorne, Phys. Rev. D 86, 074017 (2012), [arXiv:1201.6180].

[15] F. Aaron et al., [H1 and ZEUS Collaborations], Eur. Phys. J. C 73, 2311 (2013), [arXiv:1211.1182].

[16] H. Abramowicz et al. [ZEUS Collaboration], JHEP 1409, 127 (2014), [arXiv:1405.6915].

[17] F. Aaron et al. [H1 Collaboration], Eur. Phys. J. C 65, 89 (2009), [arXiv:0907.2643].

[18] J. Beringer et al. (Particle Data Group), Phys. Rev. D 86, 010001 (2012).

[19] J. Pumplin et al., JHEP 0207, 012 (2002), [hep-ph/0201195].

[20] L. Harland-Lang, A. D.Martin, P. Motylinski, and R. Thorne (2014), [arXiv:1412.3989].

[21] M. Guzzi et al., Phys. Rev. D 89, 033009 (2014), [arXiv:1302.6246].

[22] R. D. Ball et al. [NNPDF Collaboration], JHEP 1004, 040 (2015), [arXiv:1410.8849v2].

[23] S. Alekhin et al., Phys. Rev. D 86, 054009 (2012), [arXiv:1202.2281]. 